\title{
Focus. Messiaen et la technique de l'emprunt
}

Cahiers d'un compositeur récoltant et transformateur

\section{Thomas Lacôte}

\section{(2) OpenEdition}

Journals

Édition électronique

URL : https://journals.openedition.org/genesis/5737

DOI : 10.4000/genesis. 5737

ISSN : 2268-1590

Éditeur :

Presses universitaires de Paris Sorbonne (PUPS), Société internationale de génétique artistique littéraire et scientifique (SIGALES)

\section{Édition imprimée}

Date de publication : 15 décembre 2020

Pagination : 173-177

ISBN : 979-10-231-0704-3

ISSN : $1167-5101$

\section{Référence électronique}

Thomas Lacôte, « Focus. Messiaen et la technique de l'emprunt », Genesis [En ligne], 51 | 2020, mis en ligne le 20 décembre 2021, consulté le 07 février 2022. URL : http://journals.openedition.org/genesis/ 5737 ; DOI : https://doi.org/10.4000/genesis.5737

Ce document a été généré automatiquement le 7 février 2022

Tous droits réservés 


\title{
Focus. Messiaen et la technique de l'emprunt
}

\author{
Cahiers d'un compositeur récoltant et transformateur
}

\section{Thomas Lacôte}

1 Olivier Messiaen, comme d'autres compositeurs majeurs du xxe siècle, a accompagné son activité créatrice d'un important travail d'élaboration théorique. Le Traité en sept tomes issu de son enseignement - Messiaen a créé une classe d' "analyse musicale ", première du genre, au Conservatoire de Paris en 1947 - comprend plusieurs centaines de pages de commentaires, souvent de haute technicité, sur sa propre musique ${ }^{1}$. Les grands thèmes du Traité sont déjà présents dans Technique de mon langage musical ${ }^{2}$, qui joua le rôle d'introduction à la poétique de Messiaen pour plusieurs générations de compositeurs et de musicologues. C'est à partir de ces écrits que s'est constitué le savoir sur ses techniques de composition, centré sur des objets musicaux aux propriétés remarquables: échelles, rythmes, techniques de développement. La part décisive de l'intertextualité dans la genèse des œuvres de Messiaen n'a pu être révélée plus récemment que par une relecture complète de ses écrits dans une perspective nouvelle. Aujourd'hui, l'entrée des archives personnelles du compositeur à la Bibliothèque nationale de France, et plus spécialement de cahiers de travail dont des extraits sont reproduits et commentés ici pour la première fois, ouvre de nouvelles pistes pour approfondir cette recherche et démêler les fils de l'exogenèse chez Messiaen.

2 La profusion des dits et écrits de Messiaen pose un défi complexe à la génétique. Essentiellement tournés vers la désignation d'éléments de vocabulaire musicaux personnels et l'exposé de combinatoires rythmiques, les commentaires du compositeur sur son œuvre laissent de côté de vastes pans du texte musical, et gardent le silence sur les processus de création mis en œuvre. Elles recèlent des allusions intertextuelles éparses, sous formes d'analogies ${ }^{3}$, sans que ces mentions aient jusqu'à présent été considérées comme pertinentes pour la compréhension du travail créateur de Messiaen, si ce n'est comme manifestation d'une vaste culture musicale. Mes recherches et celles d'Yves Balmer et de Christopher Brent Murray ${ }^{4}$, synthétisées dans 
notre ouvrage à six mains Le modèle et l'invention, Messiaen et la technique de l'emprunt ${ }^{5}$, ont inclus une relecture complète des écrits théoriques et des œuvres de Messiaen, prenant au sérieux toutes ces allusions intertextuelles qui avaient jusqu'alors paru marginales et peu significatives. Quelles étaient exactement les sources des allusions? Quelle conscience l'auteur avait-il au moment de la création des analogies qu'elles signalent? À quelles bibliothèques et à quels imaginaires renvoyaient-elles? Quels intertextes se trouvaient au contraire passés sous silence dans les auto-analyses adressées par Messiaen à ses pairs et à ses publics?

3 Au terme de l'enquête, ces allusions sont apparues comme les indices cruciaux d'une intertextualité omniprésente, produit d'une technique très personnelle d'emprunt à un riche corpus d'œuvres du passé proche ou lointain, par ailleurs objets d'analyses musicales pratiquées par le professeur Messiaen dans sa classe au Conservatoire. Une fois collectés et transformés selon des opérations spécifiques - Messiaen les appelle son " prisme déformant ${ }^{6} »-$, ces emprunts constituent une part majeure du tissu de sa propre musique. Au-delà de la révélation d'une méthode de composition, nous avons pu reconstituer plus avant les liens qui unissent chez Messiaen le compositeur, le pédagogue, le théoricien, l'organiste, mais aussi, plus généralement, remettre sur le métier la question du modèle chez les compositeurs de la modernité musicale.

4 Cette recherche au long cours a permis de révéler un volet essentiel de la génétique musicale de Messiaen grâce à sa dimension intertextuelle : en l'absence quasi totale de documents de genèse consultables au moment de notre travail ${ }^{7}$, c'est la triangulation opérée entre les œuvres de Messiaen, ses écrits théoriques et les œuvres-sources de ses emprunts qui a permis, par accumulation d'exemples, de comprendre les constantes d'une pratique. L'intertextualité du travail créateur est ainsi devenue pour nous la clé de déchiffrement d'une génétique sans esquisses. Celle-ci emprunte ainsi dans nos travaux deux voies principales : la première s'attache à identifier, à partir du texte musical achevé, les actions de réécriture et leurs sources, et à montrer la part capitale qu'elles occupent dans le processus de création; la deuxième, à étudier ce que les écrits théoriques et les pratiques pédagogiques de compositeur peuvent entretenir de liens avec ses actions de composition. Au sein de notre corpus de travail, il faut mentionner en premier lieu l'ouvrage précédemment évoqué Technique de mon langage musical, qui peut être interprété, non seulement comme un véritable traité de transformation de l'emprunt, mais aussi, par la quantité de matériaux empruntés, transformés et " prêtsà-composer » qu'il inclut, sans toutefois les présenter comme tels, comme une esquisse à ciel ouvert.

5 Après la fin de la rédaction de notre livre et pendant la phase finale d'édition (2016-2017), le fonds Olivier Messiaen a progressivement été versé au département de la musique de la Bibliothèque nationale de France. Il représente un gigantesque ensemble d'archives documentant l'activité créatrice du compositeur, y compris le processus d'invention et ses phases préparatoires. La pratique de l'emprunt y est manifeste, et se révèle autant par l'existence de nombreuses listes de formules empruntées que par la présence conjointe sur certains feuillets des emprunts et de leur version transformée, mais surtout par d'innombrables annotations marginales que seule la technique de l'emprunt et sa permanence dans la pensée et la pratique de Messiaen permettent d'expliquer. Si une première phase d'étude de ces archives confirme l'ensemble des conclusions de notre ouvrage sur le processus créateur de Messiaen, leur exploration détaillée s'annonce comme une entreprise d'une ampleur et 
d'une complexité considérable. La brève étude proposée ci-après permet non seulement d'identifier de nouvelles sources, mais aussi d'aborder des questions en suspens : Quelle est la chronologie de la constitution de la technique de l'emprunt chez Messiaen, et son lien avec l'enseignement qu'il a reçu? Comment se constitue l'accumulation des matériaux empruntés que l'analyse des partitions laissait entrevoir? Quelle est l'amplitude réelle des sources inattendues, car n'ayant donné lieu à aucun commentaire dans les écrits et entretiens du compositeur? Comment celui-ci envisage-t-il l'identification ou la dissimulation de ses sources d'emprunts? Le fonds Messiaen constitue ainsi, non seulement un des témoignages les plus importants et les plus détaillés sur la création musicale au $\mathrm{xx}^{\mathrm{e}}$ siècle, mais aussi un levier puissant pour le réexamen des cristallisations théoriques de la modernité musicale en regard de la réalité des pratiques exogénétiques de la composition. En effet, en présentant leurs techniques de composition comme tournées vers une combinatoire interne à l'œuvre, et en leur associant parfois des appels à la rupture avec l'héritage musical, nombre de compositeurs du siècle dernier ont autant découragé toute étude d'exogenèse les concernant qu'ils ont posé les bases de commentaires théoriques, historiques et esthétiques n'ayant fait qu'amplifier par la suite cette description incomplète et biaisée des pratiques de composition.

6 Les deux pages présentées ici en fac-simile sont tirées de deux cahiers de travail, témoins privilégiés de la pratique créatrice de Messiaen : ils entremêlent en effet, sans aucune distinction nette, listes de formules empruntées, réflexions analytiques et théoriques, projets d'œuvres et esquisses à divers stades préparatoires d'élaboration, les phases finales étant réalisées sur d'autres documents.

7 La première page, tirée d'un « Cahier vert ${ }^{8}$ » (fig. 1) est datable de 1934-1935, précédant de peu La Nativité du Seigneur, pour orgue. Wagner, Stravinsky, Florent Schmitt, Ravel, Roussel, Debussy: en une seule page, c'est toute une constellation de modèles qui se constitue, dont quatre sont encore vivants à la date de rédaction de ce document. Il faut y ajouter Bach, passé par le prisme des pratiques académiques des classes de contrepoint, auquel fait allusion la mention "Faire choral (style orné) », mais aussi Marcel Dupré, compositeur et organiste, professeur de Messiaen au Conservatoire : la liste des "moyens de développement » en neuf points notée en bas de la page est en effet une citation exacte de son Traité d'improvisation à l'orgue ${ }^{9}$.

8 À ces noms de compositeur sont associés des types de préhension différents: de Wagner et Roussel, Messiaen synthétise ici des principes d'écriture, verbalisés sous la forme de notes analytiques, engageant par ce biais une forme d'abstraction quant au texte musical de référence. Mais, pour Stravinsky, Ravel, Schmitt ou Debussy, ce sont des fragments musicaux circonscrits qui sont convoqués: ces formules sont précisément l'échelle de travail privilégiée de la technique d'emprunt de Messiaen, qui sélectionne, transforme, enchâsse et homogénéise la matière même d'œuvres musicales diverses sous leur forme écrite. L'absence de localisation précise de ces fragments, inutile pour celui qui les prend ici en note, complique bien évidemment la tâche du chercheur. Les commentaires sur Debussy et Stravinsky, et dans une moindre mesure Ravel, irriguent les écrits et interviews de Messiaen. Mais il n'avait jamais été envisagé qu'un compositeur tel que Florent Schmitt ait pu constituer, à un titre ou un autre, un modèle pour Messiaen, ce dont témoignent pourtant les deux fragments collectés, qu'une enquête permet de retrouver dans son œuvre La Tragédie de Salomé (1907) ${ }^{10}$. 
9 Si la première page, sous la plume d'un jeune homme de 26 ou 27 ans, pouvait encore être assimilable à la fin d'une période d'apprentissage pour un créateur en quête de ses moyens propres, il en va tout autrement de la seconde, qui témoigne de la permanence de cette pratique de regard vers l'extérieur et de collecte organisée chez le Messiaen de la maturité, comme geste constitutif de son processus créateur. Tirée d'un «Cahier rouge $^{11}$ » utilisé au début des années 1940, cette page (fig. 2), comme bien d'autres de ce cahier, documente la genèse conjointe d'œuvres majeures comme les Visions de l'Amen (1942-1943) ou les Vingt Regards sur l'enfant Jésus (1944) et de Technique de mon langage musical, qu'il nomme à l'époque son "Traité ». Elle permet de lire différentes formules isolées, disposées sur la page comme autant de briques de construction, fragments de plus vastes carrières que sont les dictionnaires de formules générées à partir d'emprunts, dont regorge le fonds Messiaen de la BnF. Stravinsky et Debussy sont encore présents ; s'y ajoute André Jolivet, contemporain et ami de Messiaen, et source majeure d'emprunts dans les années $1940^{12}$. Sans pouvoir analyser ici la constitution, la provenance et l'emploi (souvent récurrent) dans l'œuvre de Messiaen de chacune de ces formules, il faut noter que le but de ces prélèvements n'est plus la recherche d'une technique rythmique potentiellement généralisable, comme dans la page précédente, mais plus directement celle de matériaux sélectionnés pour leur intérêt propre, agencés ensuite par diverses techniques de montage.

Fig. 1 : Olivier Messiaen, Cahier de travail dit « Cahier vert », p. 41 (ca. 1934-1935)

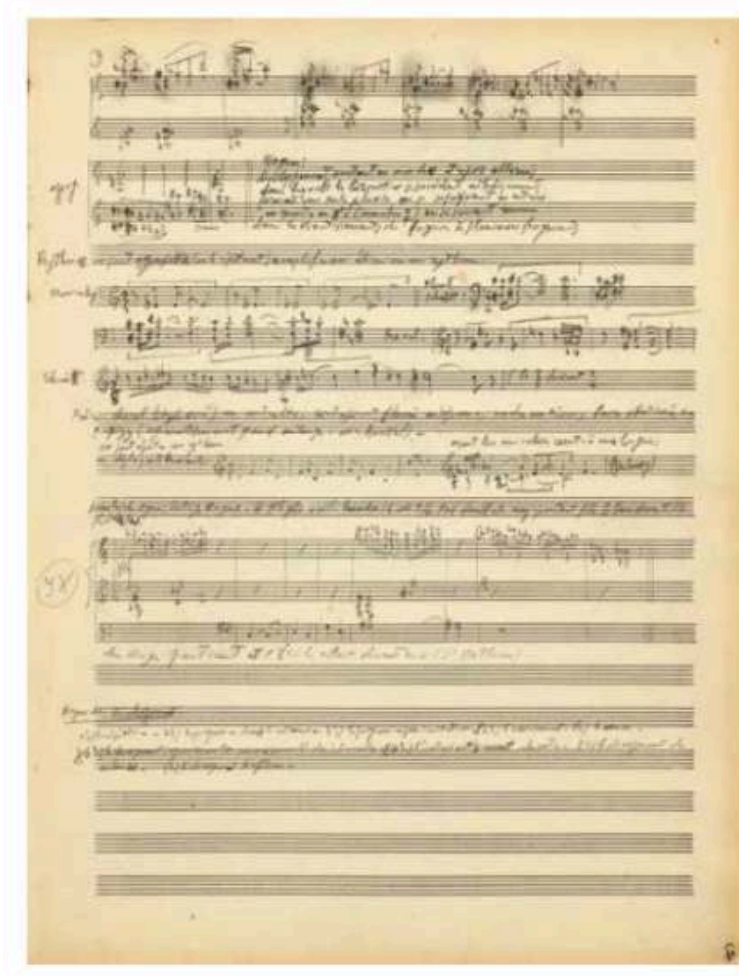

(c) Paris, BnF, Département de la musique, Ms 1491 
Fig. 2 : Olivier Messiaen, Cahier de travail dit « Cahier rouge », p. 34 (ca. 1942)

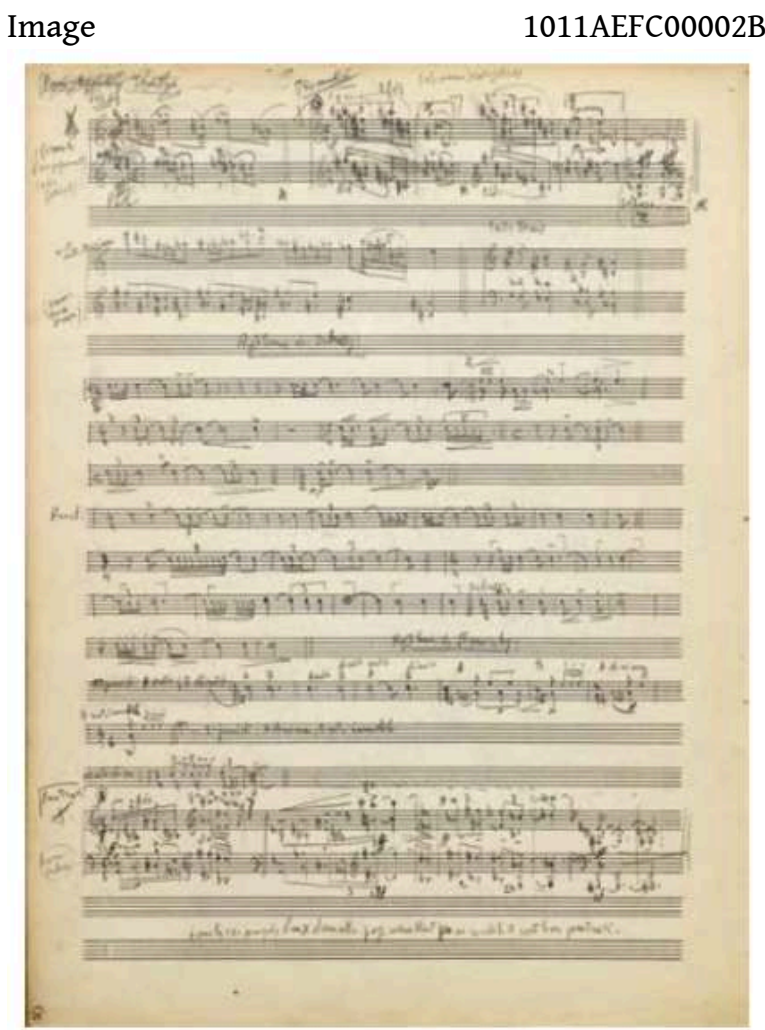

(c) Paris, BnF, Département de la musique, Ms 1492

« Tous les ex. marqués d'un X dans cette page ressemblent peu au modèle et sont bons pour Traité »: par cette phrase, Messiaen marque le succès de son entreprise de " prisme déformant ", consistant ici plus particulièrement en la présentation d'accords empruntés dans un nouveau contexte rythmique, et la nécessité pour lui d'empêcher la reconnaissance du modèle. Ces fragments pourront donc servir tout autant de bloc de construction pour une ou plusieurs œuvres, que d'exemples donnés par Messiaen de son propre «langage musical» dans son ouvrage théorique. La mention de la source sera alors soit totalement absente, soit allusive. Pour n'en donner qu'un seul exemple, la formule notée en bas de la page, annotée « harm[onie] Debussy » est un emprunt à la première des Trois Chansons de Bilitis. Devenant l'exemple 69A de Technique de mon langage musical, elle est assortie d'un seul commentaire lapidaire concernant le "caractère impressionniste » de son rythme. Mise en réserve à cette époque, elle ne fait son apparition dans l'œuvre de Messiaen qu'en 1984, dans son Livre du SaintSacrement pour orgue ${ }^{13}$. Comme à son habitude, Messiaen inclut ce fragment musical sans transposition, par montage au sein d'un tissu musical plus large, sauvegardant l'identité du morceau emprunté tout en construisant des liens de syntaxe par une technique de notes communes.

Bien que la technique de l'emprunt ne résume pas à elle seule l'exogénétique de Messiaen, elle en constitue la face la plus originale, la plus organisée, et la plus traçable dans son œuvre musicale. Plutôt que de retenir des modèles certains traits structurels ou stylistiques mis en lumière par une lecture analytique, elle s'attache à des fragments musicaux notés, localisés, tout en cherchant à leur conférer une image sonore profondément renouvelée. L'étude systématique du fonds Messiaen de la BnF 
permettra bien évidemment d'approfondir l'ampleur de cette technique. Cependant, ce bref regard sur ces deux pages montre à quel point la mise au jour des documents de genèse n'équivaut nullement à une explication complète du phénomène, tant les mentions et identifications restent lacunaires. Elles rappellent à quel point, dans l'acte d'invention musicale, la bibliothèque intérieure du compositeur peut être aussi précisément organisée que confusément enfouie. En particulier face à un siècle qui a prétendu plus que tout autre à l'oubli du passé, et malgré les possibles rétentions des compositeurs eux-mêmes, retisser les fils de l'exogenèse ne constitue pas seulement une catégorie d'approche parmi d'autres : c'est une voie majeure, pour qu'advienne une véritable connaissance de la génétique musicale, capable de ne pas réduire l'invention musicale au seul décryptage de ses opérations logiques ou à une inexplicable épiphanie.

\section{NOTES}

1. Olivier Messiaen, Traité de rythme, de couleur, et d'ornithologie (1949-2002), préface de Pierre Boulez, avant-propos d'Alain Louvier, Paris, Leduc, 1994-2002.

2. Olivier Messiaen, Technique de mon langage musical, Paris, Leduc, 1944.

3. Ces allusions prennent souvent la forme d'un "fait penser à ": "Ces accords font penser à Stravinsky ». Voir Yves Balmer, Thomas Lacôte, Christopher Brent Murray, Le modèle et l'invention, Messiaen et la technique de l'emprunt, Lyon, Symétrie, 2017, p. 552-556.

4. Yves Balmer est professeur d'analyse musicale au Conservatoire de Paris (CNSMDP) et membre permanent de l'Institut de recherche en musicologie (IReMus). Christopher Brent Murray est chercheur associé et chargé de cours à l'Université libre de Bruxelles.

5. Yves Balmer, Thomas Lacôte, Christopher Brent Murray, Le modèle et l'invention, Messiaen et la technique de l'emprunt, op. cit.

6. Voir Olivier Messiaen, Technique de mon langage musical, op. cit., p. 46.

7. Les seuls documents de ce type avec lesquels nous avons pu travailler sont les esquisses des Visions de l'Amen (1942-1943) et quelques pages d'esquisses pour La Transfiguration (1969). Voir Yves Balmer, Thomas Lacôte, Christopher Brent Murray, Le modèle et l'invention, op. cit., p. 8-9 et Yves Balmer, «Formal Genesis in the sketches for Visions de l'Amen », C. Dingle et R. Fallon (dir.), Messiaen Perspectives I: Sources and Influence, Aldershot, Ashgate, 2013, p. 69-83.

8. Olivier Messiaen, « Cahier vert », F-Pn : Rés. Vma Ms-1491.

9. Marcel Dupré, Traité d'improvisation à l'orgue, Paris, Leduc, 1925, p. 111.

10. Respectivement, chiffre 34 (mesure 3 à 5) et chiffre 50 (mesures 1 à 3 ).

11. Olivier Messiaen, « Cahier rouge », F-Pn : Rés. Vma Ms-1492.

12. Voir Yves Balmer, Thomas Lacôte, Christopher Brent Murray, Le modèle et l'invention, op. cit., p. 227-249.

13. Ibid., p. 167. 


\section{RÉSUMÉS}

Les travaux de Y.Balmer, T. Lacôte et C.Murray ont montré la place éminente que tient l'exogenèse dans le processus créateur d'olivier Messiaen, par le biais d'emprunt à un très large corpus musical et de "prisme déformant ». L'ouverture progressive du fonds Olivier Messiaen de la $\mathrm{BnF}$, riche de très nombreux documents de genèse, permet de nouvelles perspectives. Deux pages de cahier de travail commentées dans cet article montrent l'élaboration progressive de cette technique et ses modalités d'organisation.

The works of Y. Balmer, T. Lacôte and C. Murray have shown exogenesis' important place in the creative process of Olivier Messiaen, in which he borrowed from a very large corpus of known musical works to reprocess melodies through a "deforming prism". The gradual opening of the Olivier Messiaen Fund underway at the BnF, which includes numerous genetic documents, allows us new insights into his technique. In this article, two pages of his workbook throw into question both the progressive development of this technique and its organizational

Die Arbeiten von Y. Balmer, T. Lacôte und C. Murray hat den herausragenden Platz, den die Exogenese im kreativen Prozess von Olivier Messiaen einnimmt, durch Anleihen bei einem sehr großen musikalischen Korpus und ein „Verzerrungsprisma“, aufgezeigt. Die schrittweise öffnung der Olivier-Messiaen-Sammlung der Bibliothèque nationale de France, die reich an zahlreichen genetischen Dokumenten ist, eröffnet neue Perspektiven. Zwei Seiten des in diesem Artikel kommentierten Arbeitsbuches zeigen die fortschreitende Entwicklung dieser Technik und ihrer Organisationsmethoden.

Los trabajos de Y. Balmer, T. Lacôte y C. Murray han puesto de manifiesto el lugar eminente que ocupa la exogénesis en el proceso creativo de Olivier Messiaen, a través de la apropiación de un vasto corpus musical y del "prisma deformante". La apertura paulatina del fondo olivier Messiaen de la Biblioteca Nacional de Francia, que conserva numerosos documentos de génesis, abre nuevas perspectivas. Las dos páginas de un cuaderno de trabajo que comentamos en este artículo muestran la elaboración progresiva de esta técnica y de sus modalidades de organización.

Os trabalhos de Y. Balmer, T. Lacôte e C. Murray mostraram o lugar eminente que a exogénese ocupa no processo criativo de Olivier Messiaen, pela via do empréstimo a um corpus musical muito vasto e de "prisma deformante". A abertura progressiva do espólio de Olivier Messiaen da $\mathrm{BnF}$, rico em numerosos documentos de génese, permite novas perspetivas. Duas páginas de um caderno de trabalho comentadas neste artigo mostram a elaboração progressiva desta técnica e as suas modalidades de organização.

I lavori di Y. Balmer, T. Lacôte e C. Murray hanno mostrato il ruolo importante dell'esogenesi nel processo creativo di Olivier Messiaen, attraverso il prestito da un corpus musicale molto vasto e il "prisma deformante". L'apertura progressiva del fondo Olivier Messiaen della BnF, ricco di numerosi documenti genetici, apre nuove prospettive. Due pagine di un carnet di lavoro commentate in quest'articolo mostrano l'elaborazione progressiva di questa tecnica e le sue modalità d'organizzazione. 


\section{AUTEUR}

\section{THOMAS LACÔTE}

THOMAS LACôTE est titulaire du grand orgue de l'église de la Trinité. Ses activités musicales associent composition, improvisation, interprétation, pédagogie et recherche, notamment sur Olivier Messiaen. Auteur d'articles dans des revues internationales (XXth Century Music, Journal of the American Musicological Society), il a publié avec Yves Balmer et Christopher Brent Murray Le modèle et l'invention, Messiaen et la technique de l'emprunt (Lyon, Symétrie, 2017), ouvrage récompensé par le prix Musique des Muses. 